\title{
Diversities of periodic solutions for a class of ecological model
}

Yepeng Xing ${ }^{1 *}$ and Yonghui Xia ${ }^{2}$

\author{
* Correspondence: ypxing- \\ jason@hotmail.com \\ ${ }^{1}$ College of Mathematics and \\ Science, Shanghai Normal \\ University, Shanghai 200234, P.R. \\ China \\ Full list of author information is \\ available at the end of the article
}

\begin{abstract}
We consider a nonautonomous predator-prey model with nonmonotonic functional response in the periodic environment. Some new sufficient conditions are obtained for the nonexistence of periodic solutions and the global existence of at least one or two positive periodic solutions. Our method is based on Mawhin's coincidence degree and novel estimation techniques for the priori bounds of unknown solutions. Some novel estimation techniques are employed for the priori bounds of unknown solutions to $L z=\lambda N z$, which are much different from the arguments used in the previous literature. Some applications are also presented to illustrate the feasibility and effectiveness of our main results. A conclusion ends this article.

Mathematics Subject Classification (2000): Primary: 34C25; Secondary: 92D25, 92B05
\end{abstract}

Keywords: periodic solution, coincidence degree, predator-prey model, nonmonotonic functional response

\section{Introduction}

The dynamic relationship between predators and their preys has been and will continue to be one of the dominant themes in both ecology and mathematical ecology due to its universal importance [1-4]. In modelling, the different predator-prey systems, Holling [5] proposed three types of monotonic functional responses $g(x)=x, \frac{x}{m+x}, \frac{x^{2}}{m+x^{2}}$. Monotonic response functions are appropriate in many predator-prey models; however, there are experiments that indicate nonmonotonic responses occur, for example, in the cases of 'inhibition' in microbial dynamics and 'group defence' in population dynamics [6-9]. Unlike the monotonic response, the nonmonotonic response is humped and declines at high prey density $x$. Indeed, the so-called Holling type IV functional response $g(x)=\frac{x}{a+x+\frac{x^{2}}{m}}$ (which is not monotone) has been proposed and used to model the inhibitory effect at high concentrations [7]. In [8], the author investigated the following autonomous ordinary differential equation of generalized Gause type as a predator-prey model with 'group defense'.

$$
\left\{\begin{array}{l}
\frac{\mathrm{d} x}{\mathrm{~d} t}=x g(x, K)-\gamma p(x):=p(x)[F(x, K)-y] \\
\frac{\mathrm{d} y(t)}{\mathrm{d} t}=\gamma(t)[-d+q(x)] .
\end{array}\right.
$$

(c) 2012 Xing and Xia; licensee Springer. This is an Open Access article distributed under the terms of the Creative Commons Attribution License (http://creativecommons.org/licenses/by/2.0), which permits unrestricted use, distribution, and reproduction in any medium, provided the original work is properly cited. 
Taking the carrying capacity of the environment as a bifurcation parameter, they showed that the model undergoes a sequence of bifurcations include homoclinic bifurcation as well as Hopf bifurcation. Then in [9], the authors have also considered a special predator-prey model with type IV functional response.

$$
\left\{\begin{array}{l}
\frac{\mathrm{d} x(t)}{\mathrm{d} t}=r x\left[1-\frac{x(t)}{K}\right]-m \frac{x(t) y(t)}{1+b x(t)+a x^{2}(t)}, \\
\frac{\mathrm{d} y(t)}{\mathrm{d} t}=\gamma(t)\left[-d+\frac{c m x(t)}{1+b x(t)+a x^{2}(t)}\right]
\end{array}\right.
$$

where the nonmonotonic functional response is $\frac{x(t) y(t)}{1+b x(t)+a x^{2}(t)}$. They have investigated a series of bifurcations that system (1.1) undergoes, including the saddle-node bifurcation, the supercritical and subcritical Hopf bifurcations, as well as the homoclinic bifurcation. It is interesting that they found that the unique equilibrium is a cusp of codimension 3 (a degenerate Bogdanov-Takens bifurcation point). The investigation of multiple bifurcations has also been carried out on the following predator-prey system with 'group defense' using the nonmonotonic functional response $g(x)=x e^{-\beta x}$ (see [10])

$$
\left\{\begin{array}{l}
\frac{\mathrm{d} x(t)}{\mathrm{d} t}=r x\left[1-\frac{x(t)}{K}\right]-c x(t) y(t) e^{-\beta x(t)}, \\
\frac{\mathrm{d} y(t)}{\mathrm{d} t}=\gamma(t)\left[-d+\alpha x(t) e^{-\beta x(t)}\right] .
\end{array}\right.
$$

As we know, the variation of the environment plays an important role in many biological and ecological dynamic systems. To incorporate the periodicity of the environment (e.g. seasonal effects of weather, food supplies, mating habits, etc.), it is reasonable to assume periodicity of the parameters in the system. For this reason, Chen [11] has considered the following predator-prey system with Holling type IV functional response in a periodic environment

$$
\left\{\begin{array}{l}
\frac{\mathrm{d} x(t)}{\mathrm{d} t}=x(t)\left[b_{1}(t)-a_{1}(t) x\left(t-\tau_{1}(t)\right)\right]-\frac{c(t) x(t) y(t)}{\frac{x^{2}(t)}{m}+x(t)+a} \\
\frac{\mathrm{d} y(t)}{\mathrm{d} t}=\gamma(t)\left[-b_{2}(t)+\frac{a_{2}(t) x\left(t-\tau_{2}(t)\right)}{\frac{x^{2}\left(t-\tau_{2}(t)\right)}{m}+x\left(t-\tau_{2}(t)\right)+a}\right],
\end{array}\right.
$$

where $a_{i}(t), b_{i}(t)$ and $\tau_{i}(t)(i=1,2)$ are all positive periodic continuous functions with period $\omega>0$, and $m, a$ are positive real constants. By applying the method of coincidence degree and the bounds for solutions to an operator equation, some sufficient conditions have been obtained for the global existence of at least two periodic solutions of system (1.3). By employing the theory of coincidence degree and some novel estimation techniques for the priori bounds of unknown solutions to $L z=\lambda N z$, Xia et al. $[12,13]$ obtained some sufficient conditions for the discrete model and the stagestructured model with nonmonotonic functional response, respectively.

Recently, coincidence degree theory introduced by Gaines and Mawhin [14] has been a powerful tool to investigate the periodic nonautonomous systems. It has gained increasing interest in many applications to biological systems (see, e.g. [11-28]). Motivated by aforementioned discussions, in this article, we propose the following more general nonautonomous models with nonmonotonic functional response $g$ : 


$$
\left\{\begin{array}{l}
\dot{x}(t)=x(t)[a(t)-b(t) x(t)]-c(t) g(x(t)) y(t) \\
\dot{y}(t)=y(t)[-d(t)+e(t) g(x(t))]
\end{array}\right.
$$

and

$$
\left\{\begin{array}{l}
\dot{x}(t)=x(t)\left[a(t)-b(t) x\left(t-\tau_{1}(t)\right)\right]-c(t) g(x(t)) y(t) \\
\dot{y}(t)=y(t)\left[-d(t)+e(t) g\left(x\left(t-\tau_{2}(t)\right)\right)\right]
\end{array}\right.
$$

where $x(t)$ and $y(t)$ represent prey and predator densities, respectively, $a(t), b(t), c(t)$, $d(t), e(t), \tau_{1}(t)$ and $\tau_{2}(t)$ are nonnegative periodic continuous functions with period $\omega>0$. We assume the functional response $g:[0, \infty) \rightarrow[0, \infty)$ is continuous and satisfies the (NM) ('nonmonotonic') condition:

(i) $g(0)=0$;

(ii) there exists a constant $M>0$ such that $(x-M) g^{\prime}(x)<0$ for $x \neq M$.

Clearly, $g$ is increasing on $[0, M)$, decreasing on $(M, \infty)$, and $g(x) \leq g(M)$ for $x \geq 0$. Also, it is easy to see that the functions

$$
g(x)=\frac{x}{m^{2}+x^{2}}, g(x)=x e^{-\beta x} \text { and } g(x)=\frac{x}{\frac{x^{2}}{m}+x+a}
$$

appearing in (1.1)-(1.3) satisfy conditions (i)-(ii). Obviously, the systems (1.1)-(1.3) are special cases of system $(E)$ or $(F)$.

Throughout, if $f(t)$ is an $\omega$-periodic function, we shall denote

$$
\bar{f}=\frac{1}{\omega} \int_{0}^{\omega} f(t) \mathrm{d} t
$$

Clearly, $\bar{f}>0$ if $f(t)>0$, a.e. $t \in[0, \omega]$. We also set

$$
D=\frac{\bar{d}}{\bar{e}}
$$

From the point of view of mathematical biology, we choose $\mathbb{R}_{+}^{2}=\left\{(x, y)^{T} \in \mathbb{R}^{2}: x>0, y>0\right\}$ as the state space for $(E)$ and $(F)$.

We shall study the global existence of $\omega$-periodic solutions of $(E)$ and $(F)$ using $D=\frac{\bar{d}}{\bar{e}}$ as the bifurcation parameter. Some new and interesting sufficient conditions are obtained for the nonexistence of periodic solutions, global existence of at least one positive periodic solution and global existence of at least two positive periodic solutions. When system $(F)$ reduces to the particular case (1.3), our results generalize the previous results in [11]. Our method is based on Mawhin's coincidence degree and novel estimation techniques for the a priori bounds of unknown solutions. We will introduce some novel estimation techniques for the priori bounds of unknown solutions to $L z=\lambda N z$, which are much different from the arguments used in the existing literature [11-28].

The outline of the article is as follows. In Sect. 2, a sufficient condition is obtained for the nonexistence of periodic solutions. In Sect. 3, we establish criteria for the existence of at least one positive periodic solution. Section 4 is devoted to obtaining existence criteria for at least two positive periodic solutions. Finally, to illustrate the 
generality and usefulness of the results obtained, we present some applications in Sect. 5 .

\section{Nonexistence}

We shall first give a necessary condition for the existence of periodic positive solutions of the system $(E)$.

Theorem 2.1 If the system $(E)$ has a positive $\omega$-periodic solution, then $g(M) \geq D$.

Proof. We make the change of variables

$$
x(t)=\exp \{u(t)\} \text { and } \gamma(t)=\exp \{v(t)\} .
$$

Then, system $(E)$ becomes

$$
\left\{\begin{array}{l}
\dot{u}(t)=a(t)-b(t) \exp \{u(t)\}-c(t) g(\exp \{u(t)\}) \exp \{v(t)-u(t)\} \\
\dot{v}(t)=-d(t)+e(t) g(\exp \{u(t)\}) .
\end{array}\right.
$$

Obviously, system $(E)$ is equivalent to system (2.2) on $R_{+}^{2}$. Suppose the system (2.2) has a $\omega$-periodic solution $(u(t), v(t))^{T}$, i.e., $u(t+\omega)=u(t)$ and $v(t+\omega)=v(t)$. An integration of the second equation of $(2.2)$ over $[0, \omega]$ leads to

$$
v(\omega)-v(0)=\int_{0}^{\omega}[-d(t)+e(t) g(\exp \{u(t)\})] \mathrm{d} t,
$$

or

$$
0=-\bar{d} \omega+\int_{0}^{\omega} e(t) g(\exp \{u(t)\}) \mathrm{d} t .
$$

Since $g(M)$ is the maximum of $g(x)$, it follows from (2.3) that

$$
\bar{d} \omega=\int_{0}^{\omega} e(t) g(\exp \{u(t)\}) \mathrm{d} t \leq g(M) \bar{e} \omega,
$$

which implies

$$
g(M) \geq \frac{\bar{d}}{\bar{e}}=D .
$$

This completes the proof of Theorem 2.1. $\quad \square$

The following is immediate from Theorem 2.1.

Theorem 2.2 If $g(M)<D$, then the system $(E)$ has no positive $\omega$-periodic solution.

\section{Existence of one periodic solution}

In this section, we shall apply the continuation theorem of Mawhin's coincidence degree theory to establish the global existence of at least one positive periodic solution. We first summarize a few concepts from the book by Gaines and Mawhin [14].

Let $X, Y$ be real normed vector spaces. Let $L$ : Dom $L \subset X \rightarrow Y$ be a linear mapping, and $N: X \rightarrow Y$ be a continuous mapping. The mapping $L$ is called a Fredholm mapping of index zero if $\operatorname{dim} \operatorname{Ker} L=\operatorname{codim} \operatorname{Im} L<\infty$ and $\operatorname{Im} L$ is closed in $Y$. If $L$ is a Fredholm mapping of index zero, there exist continuous projectors $P: X \rightarrow X$, and $Q: Y \rightarrow Y$ such that 
$\operatorname{Im} P=\operatorname{Ker} L, \operatorname{Ker} Q=\operatorname{Im} L=\operatorname{Im}(I-Q)$. It follows that $L \mid \operatorname{Dom} L \cap \operatorname{Ker} P:(I-P) X \rightarrow \operatorname{Im} L$ is invertible. We denote the inverse of that map by $K_{p}$. If $\Omega$ is an open bounded subset of $X$, the mapping $N$ will be called L-compact on $\bar{\Omega}$ if $Q N(\bar{\Omega})$ is bounded and $K_{p}(I-Q) N: \bar{\Omega} \rightarrow X$ is compact. Since $\operatorname{Im} Q$ is isomorphic to $\operatorname{Ker} L$, there exists an isomorphism $J: \operatorname{Im} Q \rightarrow \operatorname{Ker} L$.

Lemma 3.1 (Continuation theorem [14]) Let L be a Fredholm mapping of index zero and $N$ be L-compact on $\bar{\Omega}$. Suppose

(a) for each $\lambda \in(0,1)$, every solution $z$ of $L z=\lambda N z$ is such that $z \notin \partial \Omega$;

(b) $Q N z \neq 0$ for each $z \in \partial \Omega \cap \operatorname{Ker} L$ and $\operatorname{deg}\{Q N, \Omega \cap \operatorname{Ker} L, 0\} \neq 0$.

Then, the operator equation $L z=N z$ has at least one solution lying in $\operatorname{Dom} L \cap \bar{\Omega}$.

Theorem 3.1 Assume

$\left(\mathrm{H}_{1}\right) g(M)=D$;

$\left(\mathrm{H}_{2}\right) \bar{a}>\bar{b} M \exp \{(\overline{|a|}+\bar{a}) \omega\}$.

Then, the system $(E)$ has at least one positive $\omega$-periodic solution.

Proof. We shall consider the system (2.2) (equivalent of $(E)$ ). Take

$$
X=Y=\left\{z=(u(t), v(t))^{T} \in C\left(\mathbb{R}, \mathbb{R}^{2}\right): z(t+\omega)=z(t)\right\}
$$

and define

$$
\|z\|=\max _{t \in[0, \omega]}|u(t)|+\max _{t \in[0, \omega]}|v(t)|, z=(u, v)^{T} \in X \text { or } Y,
$$

where $|\cdot|$ denotes the Euclidean norm. Then, $X$ and $Y$ are Banach spaces with the norm $\|\cdot\|$. For any $z=(u, v)^{T} \in X$, by means of the periodicity assumption, we can easily check that

$$
\begin{aligned}
& \Delta_{1}(z, t):=a(t)-b(t) \exp \{u(t)\}-c(t) g(\exp \{u(t)\}) \exp \{v(t)-u(t)\} \\
& \Delta_{2}(z, t):=-d(t)+e(t) g(\exp \{u(t)\})
\end{aligned}
$$

are $\omega$-periodic. Define $L$ on $\operatorname{Dom} L \cap X$, where $\operatorname{Dom} L=\left\{(u(t), v(t))^{T} \in C^{1}\left(\mathbb{R}, \mathbb{R}^{2}\right)\right\}$, by

$$
L\left(\begin{array}{l}
u \\
v
\end{array}\right)=\left(\begin{array}{l}
\frac{\mathrm{d} u(t)}{\mathrm{d} t} \\
\frac{\mathrm{d} v(t)}{\mathrm{d} t}
\end{array}\right)
$$

and also define $N: X \rightarrow X$ by

$$
N\left(\begin{array}{l}
u \\
v
\end{array}\right)=\left(\begin{array}{c}
\Delta_{1}(z, t) \\
\Delta_{2}(z, t)
\end{array}\right)
$$

Further, define $P$ and $Q$ on $X$ by

$$
P\left(\begin{array}{l}
u \\
v
\end{array}\right)=Q\left(\begin{array}{l}
u \\
v
\end{array}\right)=\left(\begin{array}{l}
\frac{1}{\omega} \int_{0}^{\omega} u(t) \mathrm{d} t \\
\frac{1}{\omega} \int_{0}^{\omega} v(t) \mathrm{d} t
\end{array}\right) .
$$


It is not difficult to show that

$$
\begin{gathered}
\operatorname{Ker} L=\left\{z \in X: z=C_{0}, C_{0} \in \mathbb{R}^{2}\right\} \\
\operatorname{Im} L=\left\{z \in Y: \int_{0}^{\omega} z(t) \mathrm{d} t=0\right\} \text { is closed in } Y, \\
\operatorname{dim} \operatorname{Ker} L=\operatorname{codim} \operatorname{Im} L=2,
\end{gathered}
$$

and $P$ and $Q$ are continuous projectors such that

$$
\operatorname{Im} P=\operatorname{Ker} L, \operatorname{Ker} Q=\operatorname{Im} L=\operatorname{Im}(I-Q) .
$$

It follows that $L$ is a Fredholm mapping of index zero. Furthermore, the generalized inverse (to $L$ ) $K_{p}: \operatorname{Im} L \rightarrow \operatorname{Dom} L \cap \operatorname{Ker} P$ exists, and is given by

$$
K_{p}(z)=\int_{0}^{t} z(s) \mathrm{d} s-\frac{1}{\omega} \int_{0}^{\omega} \int_{0}^{t} z(s) \mathrm{d} s \mathrm{~d} t
$$

Then, $Q N: X \rightarrow Y$ and $K_{p}(I-Q) N: X \rightarrow X$ are defined by

$$
Q N z=\left(\begin{array}{l}
\frac{1}{\omega} \int_{0}^{\omega} \Delta_{1}(z, t) \mathrm{d} t \\
\frac{1}{\omega} \int_{0}^{\omega} \Delta_{2}(z, t) \mathrm{d} t
\end{array}\right)
$$

and

$$
K_{p}(I-Q) N z=\left(\begin{array}{l}
\Psi_{1}(z, t) \\
\Psi_{2}(z, t)
\end{array}\right)
$$

where

$$
\Psi_{i}(z, t)=\int_{0}^{t} \Delta_{i}(z, s) \mathrm{d} s-\frac{1}{\omega} \int_{0}^{\omega} \int_{0}^{t} \Delta_{i}(z, s) \mathrm{d} s \mathrm{~d} t-\left(\frac{t}{\omega}-\frac{1}{2}\right) \int_{0}^{\omega} \Delta_{i}(z, s) \mathrm{d} s, i=1,2 .
$$

Clearly, $Q N$ and $K p(I-Q) N$ are continuous. Using the Arzela-Ascoli Theorem, it is not difficult to prove that $\overline{K_{p}(I-Q) N(\bar{\Omega})}$ is compact for any open bounded set $\Omega \subset X$. Moreover, $Q N(\bar{\Omega})$ is bounded. Therefore, $N$ is $L$-compact on $\bar{\Omega}$ for any open bounded set $\Omega \subset X$.

Now we need to search for an appropriate open bounded subset $\Omega$ for the application of Lemma 3.1. Corresponding to the operator equation $L z=\lambda N z$ where $\lambda \in(0,1)$, we have

$$
\left\{\begin{array}{l}
\dot{u}(t)=\lambda[a(t)-b(t) \exp \{u(t)\}-c(t) g(\exp \{u(t)\}) \exp \{v(t)-u(t)\}] \\
\dot{v}(t)=\lambda[-d(t)+e(t) g(\exp \{u(t)\})]
\end{array}\right.
$$

Suppose $z=(u(t), v(t))^{T} \in X$ is a solution of (3.1) for a certain $\lambda \in(0,1)$. An integration of (3.1) over $[0, \omega]$ leads to 


$$
\left\{\begin{array}{l}
\int_{0}^{\omega}[a(t)-b(t) \exp \{u(t)\}-c(t) g(\exp \{u(t)\}) \exp \{v(t)-u(t)\}] \mathrm{d} t=0 \\
\int_{0}^{\omega}[-d(t)+e(t) g(\exp \{u(t)\})] \mathrm{d} t=0,
\end{array}\right.
$$

or

$$
\begin{aligned}
& \int_{0}^{\omega} b(t) \exp \{u(t)\} \mathrm{d} t+\int_{0}^{\omega} c(t) g(\exp \{u(t)\}) \exp \{v(t)-u(t)\} \mathrm{d} t=\bar{a} \omega \\
& \int_{0}^{\omega} e(t) g(\exp \{u(t)\}) \mathrm{d} t=\bar{d} \omega
\end{aligned}
$$

By mean value theorem, there exists $\xi \in(0, \omega)$ such that

$$
\int_{0}^{\omega} e(t) g(\exp \{u(t)\}) \mathrm{d} t=g(\exp \{u(\xi)\}) \int_{0}^{\omega} e(t) \mathrm{d} t
$$

Hence, noting $g(M)=D=\bar{d} / \bar{e}$, it follows from (3.3) that

$$
\exp \{u(\xi)\}=M \quad \text { or } u(\xi)=\ln M .
$$

On the other hand, from the first equation of (3.1) and (3.2), we have

$$
\begin{aligned}
\int_{0}^{\omega}|\dot{u}(t)| \mathrm{d} t= & \lambda \int_{0}^{\omega} \mid a(t)-b(t) \exp \{u(t)\} \\
& -c(t) g(\exp \{u(t)\}) \exp \{v(t)-u(t)\} \mid \mathrm{d} t \\
< & \overline{|a|} \omega+\int_{0}^{\omega} b(t) \exp \{u(t)\} \mathrm{d} t \\
& +\int_{0}^{\omega} c(t) g(\exp \{u(t)\}) \exp \{v(t)-u(t)\} \mathrm{d} t \\
= & (\overline{|a|}+\bar{a}) \omega .
\end{aligned}
$$

Similarly, from the second equation of (3.1) and (3.3), it is not difficult to derive that

$$
\int_{0}^{\omega}|\dot{v}(t)| \mathrm{d} t<(\overline{|d|}+\bar{d}) \omega
$$

It follows from (3.4) and (3.5) that

$$
u(t) \leq u(\xi)+\int_{0}^{\omega}|\dot{u}(t)| \mathrm{d} t<\ln M+(\overline{|a|}+\bar{a}) \omega:=A_{1}
$$

and

$$
u(t) \geq u(\xi)-\int_{0}^{\omega}|\dot{u}(t)| \mathrm{d} t>\ln M-(\overline{|a|}+\bar{a}) \omega:=B_{1} .
$$


Since $(u(t), v(t))^{T} \in X$, there exist $\delta, \eta \in[0, \omega]$ such that

$$
v(\delta)=\min _{t \in[0, \omega]} v(t) \text { and } v(\eta)=\max _{t \in[0, \omega]} v(t) .
$$

Note that $g(M)=D$ is the maximum of $g$. Then, it follows from (3.2), (3.7)-(3.9) and $\left(H_{2}\right)$ that

$$
\bar{b} \omega \exp \left\{A_{1}\right\}+\bar{c} D \omega \exp \left\{v(\eta)-B_{1}\right\} \geq \bar{a} \omega,
$$

or

$$
v(\eta) \geq \ln \frac{\bar{a} \exp \left\{B_{1}\right\}-\bar{b} \exp \left\{A_{1}+B_{1}\right\}}{\bar{c} D}=\ln \frac{(\bar{a}-\bar{b} M \exp \{(\overline{|a|}+\bar{a}) \omega\}) M}{\bar{c} D \exp \{(\overline{|a|}+\bar{a}) \omega\}}
$$

This, combined with (3.6), gives

$$
v(t) \geq v(\eta)-\int_{0}^{\omega}|\dot{v}(t)| \mathrm{d} t>\ln \frac{(\bar{a}-\bar{b} M \exp \{(\overline{|\bar{a}|}+\bar{a}) \omega\})}{\bar{c} D \exp \{(\overline{|\bar{a}|}+\bar{a}) \omega\}}-(\overline{|d|}+\bar{d}) \omega:=A_{2} .
$$

Note that $\exp \{B 1\}<M$ and $\exp \left\{A_{1}\right\}>M$. Since $g$ is increasing on $[0, M)$ and decreasing on $(M, \infty)$, we have

$$
g(\exp \{u(t)\}) \geq \min \left\{g\left(\exp \left\{B_{1}\right\}\right), g\left(\exp \left\{A_{1}\right\}\right)\right\}, \quad u(t) \in\left(B_{1}, A_{1}\right) .
$$

This, together with (3.2), (3.7)-(3.9), leads to

$\bar{c} \omega \min \left\{g\left(\exp \left\{B_{1}\right\}\right), g\left(\exp \left\{A_{1}\right\}\right)\right\} \exp \left\{v(\delta)-A_{1}\right\} \leq \bar{a} \omega$,

or

$$
(\delta) \leq \ln \frac{\bar{a} \exp \left\{A_{1}\right\}}{\min \left\{g\left(\exp \left\{B_{1}\right\}\right), g\left(\exp \left\{A_{1}\right\}\right)\right\} \bar{c}} .
$$

Coupled with (3.6), we get

$$
v(t) \leq v(\delta)+\int_{0}^{\omega}|\dot{v}(t)| \mathrm{d} t<\ln \frac{\bar{a} \exp \left\{A_{1}\right\}}{\min \left\{g\left(\exp \left\{B_{1}\right\}\right), g\left(\exp \left\{A_{1}\right\}\right)\right\} \bar{c}}+(\overline{|d|}+\bar{d}) \omega:=B_{2} .
$$

Let

$$
A=\max \left\{\left|A_{1}\right|,\left|B_{1}\right|\right\} \text { and } B=\max \left\{\left|A_{2}\right|,\left|B_{2}\right|\right\} \text {. }
$$

Then, from (3.7), (3.8), (3.11) and (3.13), we have $|u(t)|<A$ and $|v(t)|<B$. Clearly, $A_{\dot{v}}$ $B_{i}(i=1,2)$ are independent of $\lambda$.

Now, consider the equation $Q N z=0$ where $z=(u, v)^{T} \in \mathbb{R}^{2}$, i.e.,

$$
\mathrm{QN}\left(\begin{array}{l}
u \\
v
\end{array}\right)=\left(\begin{array}{l}
\bar{a}-\bar{b} \exp \{u\}-\bar{c} g(\exp \{u\}) \exp \{v-u\} \\
-\bar{d}+\bar{e} g(\exp \{u\})
\end{array}\right)=\left(\begin{array}{l}
0 \\
0
\end{array}\right) .
$$

In view of $\left(H_{2}\right)$, we have $\bar{a}-\bar{b} M>0$. Together with $\left(H_{1}\right)$, it is easy to show that the above system has a unique solution

$$
\left(u^{*}, v^{*}\right)^{T}=\left(\ln M, \ln \frac{M \bar{e}(\bar{a}-\bar{b} M)}{\bar{c} \bar{d}}\right)^{T} .
$$


Let $C>0$ be such that

$$
\left\|\left(u^{*}, v^{*}\right)^{T}\right\|=\left|u^{*}\right|+\left|v^{*}\right|<C .
$$

Define

$$
\Omega=\{z \in X:\|z\|<A+B+C\} .
$$

It is clear that $\Omega$ satisfies condition (a) of Lemma 3.1. When $z=(u, v)^{T} \in \partial \Omega \cap \operatorname{Ker} L$ $=\partial \Omega \cap \mathbb{R}^{2}, z$ is a constant vector in $\mathbb{R}^{2}$ with $\|z\|=A+B+C$, it is clear that $Q N z \neq$ 0 . Furthermore, let $J: \operatorname{Im} Q \rightarrow \operatorname{Ker} L$ be the identity mapping. In view of the assumptions in Theorem 3.1, it is easy to see that

$$
\begin{aligned}
& \operatorname{deg}\{J Q N, \Omega \cap \operatorname{Ker} L, 0\} \\
= & \operatorname{sgn} \operatorname{det}\left[\begin{array}{cc}
W & -\bar{c} g(\exp \{u\}) \exp \{v-u\} \\
\bar{e} g^{\prime}(\exp \{u\})_{u} \exp \{u\} & 0
\end{array}\right] \\
= & \operatorname{sgn}\left\{-\overline{c e} g^{\prime}(\exp \{u\})_{u} g(\exp \{u\}) \exp \{v\}\right\} \neq 0,
\end{aligned}
$$

where $\operatorname{deg}(\cdot)$ is the Brouwer degree and

$$
W=-\bar{b} \exp \{u\}-\bar{c} g^{\prime}(\exp \{u\})_{u} \exp \{v\}+\bar{c} g(\exp \{u\}) \exp \{v-u\} \text {. }
$$

By now, we have proved that $\Omega$ satisfies all the requirements of Lemma 3.1. Thus, it follows that $L z=N z$ (i.e., system (2.2)) has at least one solution in $\operatorname{Dom} L \cap \bar{\Omega}$. The proof is complete.

\section{Existence of two periodic solutions}

In this section, we shall study system $(E)$ under the assumption $g(M)>D$. From the (NM) condition, it is easy to see that if $g(M)>D$ then the equation $g(x)=D$ has two positive solutions $r_{1}$ and $r_{2}$ such that

$$
g\left(r_{1}\right)=g\left(r_{2}\right)=D \text { and } 0<r_{1}<M<r_{2} .
$$

Theorem 4.1 Assume

$$
\begin{aligned}
& \left(H_{3}\right) g(M)>D ; \\
& \left(\mathrm{H}_{4}\right) \bar{a}>\bar{b} r_{2} \exp \{(\overline{|a|}+\bar{a}) \omega\} \\
& \left(\mathrm{H}_{5}\right) r_{1} \exp \{(\overline{|a|}+\bar{a}) \omega\} \leq M \text { and } r_{2} \geq M \exp \{(\overline{|a|}+\bar{a}) \omega\}
\end{aligned}
$$

Then, the system (E) has at least two positive $\omega$-periodic solutions.

Proof. In order to prove the existence of two periodic solutions, our most important task is to search for at least two appropriate open bounded subsets $\Omega_{1}$ and $\Omega_{2}$ in $X$ for the application of Lemma 3.1. Let $X, Y, L, N, P$ and $Q$ be defined as in the proof of Theorem 3.1, and let $z=(u(t), v(t))^{T} \in X$ be a solution of $L z=\lambda N z$ for a certain $\lambda \in$ $(0,1)$. As in the proof of Theorem 3.1, we have

$$
\begin{aligned}
& \int_{0}^{\omega} b(t) \exp \{u(t)\} \mathrm{d} t+\int_{0}^{\omega} c(t) g(\exp \{u(t)\}) \exp \{v(t)-u(t)\} \mathrm{d} t=\bar{a} \omega \\
& \int_{0}^{\omega} e(t) g(\exp \{u(t)\}) \mathrm{d} t=\bar{d} \omega
\end{aligned}
$$


and

$$
\int_{0}^{\omega}|\dot{u}(t)| \mathrm{d} t<(\overline{|a|}+\bar{a}) \omega, \quad \int_{0}^{\omega}|\dot{v}(t)| \mathrm{d} t<(\overline{|d|}+\bar{d}) \omega .
$$

Since $z=(u(t), v(t))^{T} \in X$, there exist $\varepsilon, \chi \in[0, \omega]$ such that

$$
v(\varepsilon)=\min _{t \in[0, \omega]} v(t) \text { and } v(\chi)=\max _{t \in[0, \omega]} v(t) .
$$

Note that $u(t) \neq \ln M$ for $t \in[0, \omega]$, otherwise it follows from (4.2) that $\bar{d}=\bar{e} g(M)$, which is a contradiction to $\left(H_{3}\right)$. Therefore, either $u(t) \in(-\infty, \ln M)$ or $u(t) \in(\ln M$, $\infty)$.

Case 1: $u(t)<\ln M, t \in[0, \omega]$. From (4.2), $\left(H_{3}\right)$ and the (NM) condition, there exists $\theta_{1} \in[0, \omega]$ such that

$$
\bar{d} \omega=\bar{e} \omega g\left(\exp \left\{u\left(\theta_{1}\right)\right\}\right),
$$

or

$$
\begin{aligned}
\exp \left(u\left(\theta_{1}\right)\right) & =g^{-1}(D) \in(0, M) \\
& =r_{1}
\end{aligned}
$$

This, combined with (4.3) and $\left(H_{5}\right)$, gives

$$
u(t) \leq u\left(\theta_{1}\right)+\int_{0}^{\omega}|\dot{u}(t)| \mathrm{d} t<\ln r_{1}+(\overline{|a|}+\bar{a}) \omega \leq \ln M
$$

and

$$
u(t) \geq u\left(\theta_{1}\right)-\int_{0}^{\omega}|\dot{u}(t)| \mathrm{d} t>\ln r_{1}-(\overline{|a|}+\bar{a}) \omega:=B_{1}^{*} .
$$

It follows from (4.5) and (4.6) that

$$
u(t) \in\left(B_{1}^{*}, \ln M\right) .
$$

On the other hand, from (4.1), (4.4) and (4.7), we find

$$
\bar{c} \omega g\left(\exp \left\{B_{1}^{*}\right\}\right) \exp \{v(\varepsilon)-\ln M\} \leq \bar{a} \omega,
$$

or

$$
v(\varepsilon) \leq \ln \frac{\bar{a} M}{g\left(\exp \left\{B_{1}^{*}\right\}\right) \bar{c}}
$$

This, together with (4.3), leads to

$$
v(t) \leq v(\varepsilon)+\int_{0}^{\omega}|\dot{v}(t)| \mathrm{d} t<\ln \frac{\bar{a} M}{g\left(\exp \left\{B_{1}^{*}\right\}\right) \bar{c}}+(\overline{|d|}+\bar{d}) \omega:=A_{2}^{*} .
$$

From $\left(H_{4}\right)$ and $\left(H_{5}\right)$, it is not difficult to show that $\bar{a}-\bar{b} M>0$. It follows from (4.1), (4.4) and (4.7) that 


$$
\bar{b} \omega \exp \{\ln M\}+\bar{c} \omega g(M) \exp \left\{v(\chi)-B_{1}^{*}\right\} \geq \bar{a} \omega,
$$

or

$$
v(\chi) \geq \ln \frac{(\bar{a}-\bar{b} M) \exp \left\{B_{1}^{*}\right\}}{\bar{c} g(M)}=\ln \frac{(\bar{a}-\bar{b} M) r_{1}}{\bar{c} g(M) \exp \{(\overline{|a|}+\bar{a}) \omega\}} .
$$

Coupled with (4.3), we get

$$
v(t) \geq v(\chi)-\int_{0}^{\omega}|\dot{v}(t)| \mathrm{d} t>\ln \frac{(\bar{a}-\bar{b} M) r_{1}}{\bar{c} g(M) \exp \{(\overline{|a|}+\bar{a}) \omega\}}-(\overline{|d|}+\bar{d}) \omega:=B_{2}^{*} .
$$

Therefore, from (4.9) and (4.11), we have

$$
\max _{t \in[0, \omega]}|v(t)|<\max \left\{\left|A_{2}^{*}\right|,\left|B_{2}^{*}\right|\right\}:=B^{*}, \quad \text { for } u(t) \in\left(B_{1}^{*}, \ln M\right) .
$$

Case 2: $u(t)>\ln M, t \in[0, \omega]$. From (4.2), $\left(H_{3}\right)$ and the (NM) condition, there exists $\theta_{2} \in[0, \omega]$ such that

$$
\bar{d} \omega=\bar{e} \omega g\left(\exp \left\{u\left(\theta_{2}\right)\right\}\right),
$$

or

$$
\begin{aligned}
\exp \left(u\left(\theta_{2}\right)\right)= & g^{-1}(D) \in(M, \infty) \\
& =r_{2} .
\end{aligned}
$$

This, combined with (4.3) and $\left(H_{5}\right)$, gives

$$
u(t) \leq u\left(\theta_{2}\right)+\int_{0}^{\omega}|\dot{u}(t)| \mathrm{d} t<\ln r_{2}+(\overline{|a|}+\bar{a}) \omega:=A_{1}^{+}
$$

and

$$
u(t) \geq u\left(\theta_{2}\right)-\int_{0}^{\omega}|\dot{u}(t)| \mathrm{d} t>\ln r_{2}-(\overline{|a|}+\bar{a}) \omega \geq \ln M .
$$

It follows from (4.13) and (4.14) that

$$
u(t) \in\left(\ln M, A_{1}^{+}\right) .
$$

Noting that $g(x)$ is decreasing for $x \in(M, \infty)$, it follows from (4.1), (4.4) and (4.15) that

$$
\bar{c} \omega g\left(\exp \left\{A_{1}^{+}\right\}\right) \exp \left\{v(\varepsilon)-A_{1}^{+}\right\} \leq \bar{a} \omega,
$$

or

$$
v(\varepsilon) \leq \ln \frac{\bar{a} \exp \left\{A_{1}^{+}\right\}}{g\left(\exp \left\{A_{1}^{+}\right\}\right) \bar{c}} .
$$

Together with (4.3), we get

$$
v(t) \leq v(\varepsilon)+\int_{0}^{\omega}|\dot{v}(t)| d t<\ln \frac{\bar{a} \exp \left\{A_{1}^{+}\right\}}{g\left(\exp \left\{A_{1}^{+}\right\}\right) \bar{c}}+(\overline{|d|}+\bar{d}) \omega:=A_{2}^{+} .
$$


On the other hand, it follows from (4.1), (4.4), (4.15) and condition $\left(H_{4}\right)$ that

$$
\bar{b} \omega \exp \left\{A_{1}^{+}\right\}+\bar{c} \omega g(M) \exp \{v(\chi)-\ln M\} \geq \bar{a} \omega
$$

or

$$
v(\chi) \geq \ln \frac{\left(\bar{a}-\bar{b} \exp \left\{A_{1}^{+}\right\}\right) M}{\bar{c} g(M)}=\ln \frac{\left(\bar{a}-\bar{b} r_{2} \exp \{(\overline{|a|}+\bar{a}) \omega\}\right) M}{\bar{c} g(M)} .
$$

This, combined with (4.3), gives

$$
v(t) \geq v(\chi)-\int_{0}^{\omega}|\dot{v}(t)| d t>\ln \frac{\left(\bar{a}-\bar{b} \exp \left\{A_{1}^{+}\right\}\right) M}{\bar{c} g(M)}-(\overline{|d|}+\bar{d}) \omega:=B_{2}^{+} .
$$

Therefore, from (4.17) and (4.19), we get

$$
\max _{t \in[0, \omega]}|v(t)|<\max \left\{\left|A_{2}^{+}\right|,\left|B_{2}^{+}\right|\right\}:=B^{+}, \quad \text { for } u(t) \in\left(\ln M, A_{1}^{+}\right) .
$$

Obviously, $r_{1}, r_{2}, \ln M, B_{1}^{*}, A_{1}^{+}, B^{*}$ and $B^{+}$are independent of $\lambda$.

Now, let us consider the equation $Q N z=0$ where $z=(u, v)^{T} \in \mathbb{R}^{2}$, i.e., (3.14). Noting the (NM) condition, $\left(H_{3}\right)$ and $\left(H_{4}\right)$, it is not difficult to show that (3.14) has two distinct solutions

$$
(\tilde{u}, \tilde{v})^{T}=\left(\ln r_{1}, \ln \left\{\frac{\left(\bar{a}-\bar{b} r_{1}\right) \bar{e} r_{1}}{\bar{c} \bar{d}}\right\}\right)
$$

and

$$
(\hat{u}, \hat{v})^{T}=\left(\ln r_{2}, \ln \left\{\frac{\left(\bar{a}-\bar{b} r_{2}\right) \bar{e} r_{2}}{\bar{c} \bar{d}}\right\}\right) .
$$

Choose $C_{0}>0$ such that

$$
\max \left\{\left|\ln \left\{\frac{\left(\bar{a}-\bar{b} r_{1}\right) \bar{e} r_{1}}{\bar{c} \bar{d}}\right\}\right|,\left|\ln \left\{\frac{\left(\bar{a}-\bar{b} r_{2}\right) \bar{e} r_{2}}{\bar{c} \bar{d}}\right\}\right|\right\}<C_{0} .
$$

Define

$$
\Omega_{1}=\left\{z=(u, v)^{T} \in X: u(t) \in\left(B_{1}^{*}, \ln M\right), \max _{t \in[0, \omega]}|v(t)|<B^{*}+C_{0}\right\}
$$

and

$$
\Omega_{2}=\left\{x=(u, v)^{T} \in X: u(t) \in\left(\ln M, A_{1}^{+}\right), \max _{t \in[0, \omega]}|v(t)|<B^{+}+C_{0}\right\} .
$$

Both $\Omega_{1} \Omega_{2}$ are bounded open subsets of $X$. It follows from the (NM) condition, (4.12) and (4.20) that $(\tilde{u}, \tilde{v})^{T} \in \Omega_{1}$ and $(\hat{u}, \hat{v})^{T} \in \Omega_{2}$. In view of (4.12) and (4.20), it is easy to see that $\Omega_{1} \cap \Omega_{2}=\varnothing$ and $\Omega_{i}$ satisfies condition (a) of Lemma 3.1 for $i=1,2$. Moreover, $Q N z \neq 0$ for $z \in \partial \Omega_{i} \cap \operatorname{Ker} L=\partial \Omega_{i} \cap \mathbb{R}^{2}$. A direct computation gives 


$$
\begin{aligned}
\operatorname{deg}\left\{J Q N, \Omega_{i} \cap \operatorname{Ker} L, 0\right\} & =\operatorname{sgn}\left\{-\bar{c} \bar{e} g^{\prime}(\exp \{u\})_{u} g(\exp \{u\}) \exp \{v\}\right\} \\
& =(-1)^{i+1} \neq 0 .
\end{aligned}
$$

Here, $J$ is taken as the identity mapping since $\operatorname{Im} Q=\operatorname{Ker} L$. We have proved that $\Omega_{i}$ satisfies all the conditions in Lemma 3.1. Hence, $L z=N z$ (i.e., system (2.2)) has at least two $\omega$-periodic solutions $z^{*}=\left(u^{*}, v^{*}\right)^{T}$ and $z^{+}=\left(u^{+}, v^{+}\right)^{T}$ with $z^{*} \in \operatorname{Dom} L \cap \bar{\Omega}_{1}$ and $z^{+} \in \operatorname{Dom} L \cap \bar{\Omega}_{2}$.

Obviously, $z^{*}$ and $z^{+}$are different. Using (2.1),

$$
\left(\exp \left\{u^{*}(t)\right\}, \exp \left\{v^{*}(t)\right\}\right)^{T} \text { and }\left(\exp \left\{u^{+}(t)\right\}, \exp \left\{v^{+}(t)\right\}\right)^{T}
$$

are two different positive $\omega$-periodic solutions of $(E)$. This ends the proof. Box

We are now ready to tackle the system $(F)$. With the change of variables in (2.1), the system $(F)$ becomes

$$
\left\{\begin{array}{l}
\dot{u}(t)=a(t)-b(t) \exp \left\{u\left(t-\tau_{1}(t)\right)\right\}-c(t) g(\exp \{u(t)\}) \exp \{v(t)-u(t)\} \\
\dot{v}(t)=-d(t)+e(t) g\left(\exp \left\{u\left(t-\tau_{2}(t)\right)\right\}\right) .
\end{array}\right.
$$

The arguments used in the proof of Theorem 2.1 will still be valid. As for the analog of Theorem 3.1, corresponding to (3.3) we have

$$
\int_{0}^{\omega} e(t) g\left(\exp \left\{u\left(t-\tau_{2}(t)\right)\right\}\right) \mathrm{d} t=\bar{d} \omega .
$$

By mean value theorem, there exists $\xi_{0} \in(0, \omega)$ such that

$$
\int_{0}^{\omega} e(t) g\left(\exp \left\{u\left(t-\tau_{2}(t)\right)\right\}\right) \mathrm{d} t=g\left(\exp \left\{u\left(\xi_{0}-\tau_{2}\left(\xi_{0}\right)\right)\right\}\right) \int_{0}^{\omega} e(t) \mathrm{d} t .
$$

Since $u$ is $\omega$-periodic, there exists $\xi \in[0, \omega]$ such that

$$
u\left(\xi_{0}-\tau_{2}\left(\xi_{0}\right)\right)=u(\xi) .
$$

Thus, we have

$$
\int_{0}^{\omega} e(t) g\left(\exp \left\{u\left(t-\tau_{2}(t)\right)\right\}\right) \mathrm{d} t=g(\exp \{u(\xi)\}) \int_{0}^{\omega} e(t) \mathrm{d} t
$$

and the rest of the arguments in the proof of Theorem 3.1 follows. We have the following result for the system $(F)$.

Theorem 4.2 Theorems 2.1, 2.2, 3.1, 4.1 are also valid for the delayed system (F).

Remark 4.1 We have introduced some novel estimation techniques for the priori bounds of unknown solutions to $L z=\lambda \mathrm{Nz}$, which are much different from the arguments used in [11-27]. In addition, we study the diversity of the periodic solutions including nonexistence of periodic solutions, at least one periodic solutions and at least two periodic solutions. However, the existing literature only gave some sufficient conditions for one of the three cases.

\section{Applications}

In this section, we shall list some applications of Theorems 2.1, 2.2, 3.1, 4.1 and 4.2. 


\section{Application 5.1}

Consider the following nonautonomous periodic system [11]

$$
\left\{\begin{array}{l}
\frac{d x(t)}{d t}=x(t)\left[b_{1}(t)-a_{1}(t) x\left(t-\tau_{1}(t)\right)\right]-\frac{c(t) x(t) y(t)}{\frac{x^{2}(t)}{m}+x(t)+a} \\
\frac{d y(t)}{d t}=y(t)\left[-b_{2}(t)+\frac{a_{2}(t) x\left(t-\tau_{2}(t)\right)}{\frac{x^{2}\left(t-\tau_{2}(t)\right)}{m}+x\left(t-\tau_{2}(t)\right)+a}\right]
\end{array}\right.
$$

where

$$
g(x)=\frac{x}{\frac{x^{2}}{m}+x+a} .
$$

Note that $g$ satisfies the (NM) condition. It is easy to show that

$$
g^{\prime}(x)=\frac{a-\frac{x^{2}}{m}}{\left(\frac{x^{2}}{m}+x+a\right)^{2}} .
$$

Thus, $g^{\prime}(M)=0$ provides $M=\sqrt{a m}$, and

$$
g(M)=g(\sqrt{a m})=\frac{\sqrt{a m}}{2 a+\sqrt{a m}} .
$$

Corresponding to the system $(F)$, we have

$$
\bar{a}=\overline{b_{1}}, \bar{b}=\overline{a_{1}}, \bar{c}=\bar{c}, \bar{d}=\overline{b_{2}}, \bar{e}=\overline{a_{2}} .
$$

Solving $g(x)=D=\frac{\bar{d}}{\bar{e}}$ yields

$$
r_{1}=\frac{m\left(\overline{a_{2}}-\overline{b_{2}}\right)-m \sqrt{\Delta}}{2 \overline{b_{2}}} \text { and } r_{2}=\frac{m\left(\overline{a_{2}}-\overline{b_{2}}\right)+m \sqrt{\Delta}}{2 \overline{b_{2}}}
$$

where

$$
\Delta=\left(\overline{a_{2}}-\overline{b_{2}}\right)^{2}-4{\overline{b_{2}}}^{2} \frac{a}{m} .
$$

Applying Theorem 4.2 to the system (5.1), we obtain the following results.

Theorem 5.1 If $\overline{a_{2}}<\overline{b_{2}}\left(1+2 \sqrt{\frac{a}{m}}\right)$, then the system (5.1) has no positive $\omega$-periodic solution.

Theorem 5.2 Assume

$\left(I_{1}\right) \overline{a_{2}}=\overline{b_{2}}\left(1+2 \sqrt{\frac{a}{m}}\right)$;

$\left(I_{2}\right) \overline{b_{1}}>\overline{a_{1}} \sqrt{a m} \exp \left\{\left(\overline{\left|b_{1}\right|}+\overline{b_{1}}\right) \omega\right\}$.

Then, the system (5.1) has at least one positive $\omega$-periodic solution.

Theorem 5.3 Assume

$\left(I_{3}\right) \overline{a_{2}}>\overline{b_{2}}\left(1+2 \sqrt{\frac{a}{m}}\right)$;

(I $\left.I_{4}\right) \overline{b_{1}}>\overline{a_{1}} r_{2} \exp \left\{\left(\overline{\left|b_{1}\right|}+\overline{b_{1}}\right) \omega\right\}$;

$\left(I_{5}\right) r_{1} \exp \left\{\left(\overline{\left|b_{1}\right|}+\overline{b_{1}}\right) \omega\right\} \leq \sqrt{a m}$ and $r_{2} \geq \sqrt{a m} \exp \left\{\left(\overline{\left|b_{1}\right|}+\overline{b_{1}}\right) \omega\right\}$; 
where $r_{1}$ and $r_{2}$ are given in (5.2). Then, the system (5.1) has at least two positive $\omega$ periodic solutions.

Remark 5.1 In [11], the author has studied the system (5.1) and obtained the following theorem:

Theorem A. Assume

(i) $\overline{a_{2}}>\overline{b_{2}}\left(1+2 \sqrt{\frac{a}{m}}\right) \exp \left\{\left(\overline{\left|b_{1}\right|}+\overline{b_{1}}\right) \omega\right\}$;

(ii) $\overline{b_{1}}>\overline{a_{1}} l_{+} \exp \left\{\left(\overline{\left|b_{1}\right|}+\overline{b_{1}}\right) \omega\right\}$, where

$$
l_{+}=\frac{m\left[\bar{a}_{2} \exp \left\{\left(\left|\overline{b_{1}}\right|+\overline{b_{1}}\right) \omega\right\}-\overline{b_{2}}\right]+m \sqrt{\left[\overline{a_{2}} \exp \left\{\left(\left|\overline{b_{1}}\right|+\overline{b_{1}}\right) \omega\right\}-\overline{b_{2}}\right]^{2}-4{\overline{b_{2}}}^{2} a / m}}{2 \overline{b_{2}}} .
$$

Then, the system (5.1) has at least two positive $\omega$-periodic solutions.

Obviously, the conditions $\left(I_{3}\right)$ and $\left(I_{4}\right)$ in Theorem 5.3 are much weaker than the conditions (i) and (ii) in Theorem A. Certainly, we also need the condition $\left(I_{5}\right)$. Note that our novel estimation techniques for the a priori bounds of unknown solutions to $L z=\lambda N z$ are much different from those used in [11]. Moreover, there are no similar results to Theorems 5.1 and 5.2 in [11].

\section{Application 5.2}

Consider the following nonautonomous periodic system

$$
\left\{\begin{array}{l}
\frac{\mathrm{d} x(t)}{\mathrm{d} t}=r(t) x(t)\left[1-\frac{x(t)}{K(t)}\right]-\frac{x(t) y(t)}{m^{2}+x^{2}(t)} \\
\frac{\mathrm{d} y(t)}{\mathrm{d} t}=\gamma(t)\left[-d(t)+\frac{\mu(t) x(t)}{m^{2}+x^{2}(t)}\right]
\end{array}\right.
$$

where

$$
g(x)=\frac{x}{m^{2}+x^{2}} .
$$

When $r(t) \equiv r, \mu(t) \equiv \mu$ and $d(t) \equiv d \alpha(t) \equiv \alpha$, the system (5.3) reduces to one which has been studied by Chen and Zhou [29]. Note that $g$ satisfies the (NM) condition. Solving $g^{\prime}(M)=0$ gives $M=m$, and $g(M)=g(m)=\frac{1}{2 m}$. Corresponding to the system $(E)$, we have

$$
\bar{a}=\bar{r}, \bar{b}=\overline{\left(\frac{r}{K}\right)}, c=1, \bar{d}=\bar{d}, \bar{e}=\bar{\mu} .
$$

Solving $g(x)=D=\frac{\bar{d}}{\bar{e}}$ yields

$$
r_{1}=\frac{\bar{\mu}-\sqrt{\bar{\mu}^{2}-4 \bar{d}^{2} m^{2}}}{2 \bar{d}} \text { and } r_{2}=\frac{\bar{\mu}+\sqrt{\bar{\mu}^{2}-4 \bar{d}^{2} m^{2}}}{2 \bar{d}} .
$$

Applying our results to the system (5.3), we have the following theorems.

Theorem 5.4 If $\frac{1}{2 m}<\frac{\bar{d}}{\bar{\mu}}$, then the system (5.3) has no positive w-periodic solution.

Theorem 5.5 Assume

$\left(J_{1}\right) \frac{1}{2 m}=\frac{\bar{d}}{\bar{\mu}}$; 
$\left(J_{2}\right) \bar{r}>\overline{\left(\frac{r}{K}\right)} m \exp \{(\overline{|r|}+\bar{r}) \omega\}$.

Then, the system (5.3) has at least one positive $\omega$-periodic solution.

Theorem 5.6 Assume

(J) $\frac{1}{2 m}>\frac{\bar{d}}{\bar{\mu}}$;

(J) $2 \bar{d} \bar{r}>\overline{\left(\frac{r}{K}\right)}\left(\bar{\mu}+\sqrt{\bar{\mu}^{2}-4 \bar{d}^{2} m^{2}}\right) \exp \{(\overline{|r|}+\bar{r}) \omega\} ;$

$\left(J_{5}\right)$

$$
\left(\bar{\mu}-\sqrt{\bar{\mu}^{2}-4 \bar{d}^{2} m^{2}}\right) \exp \{(\overline{|r|}+\bar{r}) \omega\} \leq 2 m \bar{d}
$$

and

$\left(\bar{\mu}+\sqrt{\bar{\mu}^{2}-4 \bar{d}^{2} m^{2}}\right) \geq 2 m \bar{d} \exp \{(\overline{|r|}+\bar{r}) \omega\}$.

Then, the system (5.3) has at least two positive w-periodic solutions.

\section{Application 5.3}

Consider the following nonautonomous periodic system

$$
\left\{\begin{array}{l}
\frac{\mathrm{d} x(t)}{\mathrm{d} t}=r(t) x(t)\left[1-\frac{x(t)}{K(t)}\right]-c(t) x(t) y(t) e^{-\beta x(t)}, \\
\frac{\mathrm{d} y(t)}{\mathrm{d} t}=\gamma(t)\left[-d(t)+\alpha(t) x(t) e^{-\beta x(t)}\right]
\end{array}\right.
$$

where

$$
g(x)=x e^{-\beta x} .
$$

Note that $g$ satisfies the (NM) condition. When $r(t) \equiv r, c(t) \equiv c, d(t) \equiv d$ and $\alpha(t)=$ $\alpha$, the system (5.5) reduces to one which has been studied by [10].

Obviously, $g^{\prime}(M)=0$ gives $M=\frac{1}{\beta}$, and $g(M)=\frac{1}{\beta e}$. Corresponding to the system $(E)$, we have

$$
\bar{a}=\bar{r}, \quad \bar{b}=\overline{\left(\frac{r}{K}\right)}, \quad \bar{c}=\bar{c}, \quad \bar{d}=\bar{d}, \quad \bar{e}=\bar{\alpha} .
$$

The equation $g(x)=D=\overline{\bar{d}}$ is equivalent to

$$
F(x)=x e^{-\beta x}-\frac{\bar{d}}{\bar{\alpha}}=0 .
$$

If $g(M)>\frac{\bar{d}}{\bar{\alpha}}$, then it is easy to show that $F(0)<0, F(M)>0$ and $F(\infty)<0$. Thus, there exist $r_{1} \in(0, M)$ and $r_{2} \in(M, \infty)$ such that

$$
F\left(r_{1}\right)=0 \text { and } F\left(r_{2}\right)=0 \text {. }
$$

Applying our results to the system (5.5), we have the following theorems.

Theorem 5.7 If $\frac{1}{\beta e}<\frac{\bar{d}}{\bar{\alpha}}$, then the system (5.5) has no positive $\omega$-periodic solution.

Theorem 5.8 Assume

$\left(K_{1}\right) \frac{1}{\beta e}=\frac{\bar{d}}{\bar{\alpha}} ;$

$\left(K_{2}\right) \bar{r}>\overline{\left(\frac{r}{K}\right)} \frac{1}{\beta} \exp \{(\overline{|r|}+\bar{r}) \omega\}$.

Then, the system (5.5) has at least one positive $\omega$-periodic solution. 


\section{Theorem 5.9 Assume}

$\left(K_{3}\right) \frac{1}{\beta e}>\frac{\bar{d}}{\bar{\alpha}}$

(K $2 \bar{r}>\overline{\left(\frac{r}{K}\right)} r_{2} \exp \{(\overline{|r|}+\bar{r}) \omega\}$

$\left(K_{5}\right) r_{1} \exp \{(\overline{|r|}+\bar{r}) \omega\} \leq \frac{1}{\beta}$ and $r_{2} \geq \frac{1}{\beta} \exp \{(\overline{|r|}+\bar{r}) \omega\}$

where $r_{1}$ and $r_{2}$ are given in (5.6). Then, the system (5.5) has at least two positive $\omega$ periodic solutions.

\section{Conclusion}

A class of nonautonomous predator-prey model with nonmonotonic functional response in this paper. Some new sufficient conditions are obtained for the nonexistence of periodic solutions and the global existence of at least one or two positive periodic solutions. Our method is based on Mawhin's coincidence degree and novel estimation techniques for the priori bounds of unknown solutions. Some novel estimation techniques are employed for the priori bounds of unknown solutions to $L z=\lambda N z$, which are much different from the arguments used in the previous literature. Some applications are also presented to illustrate the feasibility and effectiveness of our main results.

\section{Acknowledgements}

This work was supported by the National Natural Science Foundation of China under Grant (No.10971139), Shanghai Municipal Educational Committee (No.10YZ74), Natural Science Foundation of Shanghai (No.12ZR1421600), National Natural Science Foundation of China (No.10901140) and ZJNSFC (No.Y6100696).

\section{Author details}

${ }^{1}$ College of Mathematics and Science, Shanghai Normal University, Shanghai 200234, P.R. China ${ }^{2}$ Department of Mathematics, Zhejiang Normal University, Jinhua 321004, China

Authors' contributions

All authors read and approved the final manuscript.

\section{Competing interests}

The authors declare that they have no competing interests.

Received: 29 December 2011 Accepted: 16 July 2012 Published: 16 July 2012

\section{References}

1. Bazykin, AD: Nonlinear Dynamics of Interacting Populations. World Scientific, Singapore (1998)

2. Berryman, AA: The origins and evolution of predator-prey theory. Ecology. 75, 1530-1535 (1992)

3. Freedman, HI: Deterministic Mathematical Models in Population Ecology. Marcel Dekker, New York (1980)

4. Kot, M: Elements of Mathematical Ecology. Cambridge University Press, New York (2001)

5. Holling, CS: The functional response of predator to prey density and its role in mimicry and population regulation. Men Entomol Soc Can. 45, 1-60 (1965)

6. Bush, AW, Cook, AE: The effect of time delay and growth rate inhibition in the bacterial treatment of wastewater. J Theor Biol. 63, 385-395 (1976). doi:10.1016/0022-5193(76)90041-2

7. Lundberg, P, Fryxell, JM: Expected population density versus productivty in ratio-dependent and prey-dependent models. Am Nat. 145, 153-161 (1995)

8. Wolkowicz, GSK: Bifurcation analysis of a predator-prey system involving group defence. SIAM J Appl Math. 48, 592-606 (1988). doi:10.1137/0148033

9. Zhu, HP, Campbell, SA, Wolkowicz, GSK: Bifurcation analysis of a predator-prey system with nonmonotonic function response. SIAM J Appl Math. 63(2), 636-682 (2002)

10. Xiao, D, Ruan, S: Codimension two bifurcations in a delayed predator-prey system with group defense. Int J Bifurc Chaos. 11(8), 2123-2132 (2001). doi:10.1142/S021812740100336X

11. Chen, Y: Multiple periodic solution of delayd predator-prey systems with type IV functional responses. Nonliear Anal RWA. 5, 45-53 (2004). doi:10.1016/S1468-1218(03)00014-2

12. Xia, YH, Cao, J, Lin, M: Discrete-time analogues of predator-prey models with monotonic or nonmonotonic functional responses. Nonlinear Anal RWA. 8(4), 1079-1095 (2007). doi:10.1016/j.nonrwa.2006.06.007

13. Xia, YH, Cao, J, Cheng, SS: Multiple periodic solutions of a delayed stage-structured predator-prey model with nonmonotone functional responses. Appl Math Model. 31(9), 1947-1959 (2007). doi:10.1016/j.apm.2006.08.012

14. Gaines, RE, Mawhin, JL: Coincidence Degree and Nonlinear Differential Equations. Springer, Berlin (1977) 
15. Xia, YH, Han, M: New conditions on the existence and stability of periodic solution in Lotka-Volterra's population system. SIAM J Appl Math. 69(6), 1580-1597 (2009). doi:10.1137/070702485

16. Fan, M, Wang, Q, Zou, XF: Dynamics of a nonautonomous ratio-dependent predator-prey system. Proc R Soc A. 133, $97-118(2003)$

17. Kuang, Y: Delay Differential Equations With Application Dynamic. Academic Press, New York (1988)

18. Gopalsamy, K: Stability and oscillation in delay differential equations of population dynamics. In Mathematics and its Applications, vol. 74,Kluwer, Dordrecht (1992)

19. Fan, Y, Li, WT, Wang, L: Periodic solutions of delayed ratio-dependent predator-prey models with monotonic or nonmonotonic fucntional responses. Nonlinear Anal. 5, 247-263 (2004). doi:10.1016/S1468-1218(03)00036-1

20. Jin, Z, Ma, Z: Stability for a competitive Lotka-Volterra system with delays. Chaos Solitons Fractals. 26, 559-569 (2005). doi:10.1016/j.chaos.2005.01.064

21. Jin, Z, Ma, Z: Periodic solutions for delay differential equations model of plankton allelopathy. Comput Math Appl. 44, 491-500 (2002). doi:10.1016/S0898-1221(02)00163-3

22. Song, QK, Zhang, J: Global exponential stability of impulsive Cohen-Grossberg neural network with time-varying delays. Nonlinear Anal RWA. 9(2), 500-510 (2008). doi:10.1016/j.nonrwa.2006.11.015

23. Song, QK, Wang, ZD: Stability analysis of impulsive stochastic CohenCGrossberg neural networks with mixed time delays. Physica A. 387, 3314-3326 (2008). doi:10.1016/j.physa.2008.01.079

24. Xia, YH, Cao, J, Han, M: A new analytical method for the linearization of dynamic equation on measure chains. J Differ Equ. 235(2), 527-543 (2007). doi:10.1016/j.jde.2007.01.004

25. Xia, YH, Han, M, Huang, ZK: Global attractivity of an almost periodic N-species nonlinear ecological competitive model. J Math Anal Appl. 337, 144-168 (2008). doi:10.1016/j.jmaa.2007.03.103

26. Ding, W, Han, M: Dynamic of a non-autonomous predator-prey system with infinite delay and diffusion. Comput Math Appl. 56, 1335-1350 (2008). doi:10.1016/j.camwa.2008.03.001

27. Freedman, HI, Kuang, Y: Stability switches in linear scalar neutral delay equations. Funkc Ekvac. 34, 187-209 (1991)

28. Chen, L: Mathematical Models and Methods in Ecology. Science Press, Bejing (1998) (in Chinese)

29. Chen, G, Zhou, J: Boundary Element Methods. Academic Press, London (1992)

doi:10.1186/1687-1847-2012-108

Cite this article as: Xing and Xia: Diversities of periodic solutions for a class of ecological model. Advances in

Difference Equations 2012 2012:108.

\section{Submit your manuscript to a SpringerOpen ${ }^{\circ}$} journal and benefit from:

- Convenient online submission

- Rigorous peer review

- Immediate publication on acceptance

- Open access: articles freely available online

- High visibility within the field

Retaining the copyright to your article

Submit your next manuscript at $\gg$ springeropen.com 